\title{
An Analytical Optimisation Framework for Airport Terminal Capacity Expansion
}

\author{
Sultan Alodhaibi $\left(\mathbb{D},{ }^{1}\right.$ Robert L. Burdett, ${ }^{2}$ and Prasad K. D. V. Yarlagadda ${ }^{3}$ \\ ${ }^{1}$ Department of Mathematics, College of Sciences and Arts in Al-Rass, Qassim University, Buraidah 51921, Saudi Arabia \\ ${ }^{2}$ School of Mathematical Science, Science and Engineering Faculty, Queensland University of Technology, Brisbane, \\ QLD 4001, Australia \\ ${ }^{3}$ School of Mechanical Medical and Process Engineering, Science and Engineering Faculty, Queensland University of Technology, \\ Brisbane, QLD 4001, Australia
}

Correspondence should be addressed to Sultan Alodhaibi; sathaieby@qu.edu.sa

Received 5 May 2020; Revised 26 June 2020; Accepted 29 July 2020; Published 5 October 2020

Guest Editor: Baogui Xin

Copyright (C) 2020 Sultan Alodhaibi et al. This is an open access article distributed under the Creative Commons Attribution License, which permits unrestricted use, distribution, and reproduction in any medium, provided the original work is properly cited.

\begin{abstract}
This article considers how to allocate additional physical resources within airport terminals. An optimization model was developed to determine where additional resources should be placed to minimise passenger waiting times. The objective function is stochastic and can only be evaluated using discrete event simulation. As this model is stochastic and nonlinear, a Simulated Annealing (SA) metaheuristic was implemented and tested. The SA algorithm repeatedly perturbs a resource allocation solution using one of two methods. The first method is creating new solution randomly in each iteration, and the second method is local search that is mimicked by any move of the current solution of $x$ solution chosen randomly in its neighborhood. Numerical testing shows that the random approach is best, and solutions that are $12.11 \%$ better can be obtained.
\end{abstract}

\section{Introduction}

The high growth in passenger numbers in recent years has created considerable strains on airports. Airport terminals are expected to process the increasing passenger numbers efficiently and with minimum delay. At the same time, the required expansion of the airport capacity might be limited by the available resources (e.g., limited available land), environmental impacts, and lengthy approval processes [1]. In addition, extension of the major airport infrastructure is typically time-consuming and costly, which raises the need for the development of smart systems and methods to improve airport performance within the available infrastructure.

Airport terminals are complex systems and are inherently stochastic in nature. Passenger numbers continually change throughout the day, depending on the status of incoming or outgoing flights. It is an integrated system, and operational problems within any of its internal processes can jeopardise the performance of other elements, creating significant bottlenecks, long passenger queues, congestion, and overall delays [2-4]. For example, disruption, congestion, and uneven passenger inflow into the terminal processing points, caused by the operation of the landside element (including the infrastructure and facilities associated with the arrival of passengers to the airport), could have a significant impact on the performance of the terminal (such as passenger boarding and take-off procedures). It involves multiple stakeholders, and each is responsible for performing particular terminal process such as check-in, security, and immigration [5].

It is conventional to subdivide airport operations into those relevant to the arrival procedures of incoming passengers and departure procedures for outgoing passengers. The arrival processes and facilities include disembarking, immigration, baggage claim, and quarantine procedures [6]. The departure processes and facilities include check-in, security screening, immigration and customs, boarding, and 
take-off procedures. It is these departure flow processes that have the greatest impact on the entire operation of passenger terminals and other elements of the airport. According to Neufville and Odoni [7], the departure process requires significantly more time than the arrival process because it sometimes involves services provided to transit passengers. Due to the complex structure of airport terminals, the development of an analytical optimization framework for studying passengers flow in airports under uncertainty of future demand is a difficult task. These difficulties and challenges have led to studies of overall terminal capacity planning problems. Previous studies have generally focused on one element of the terminal or have not accounted for expandability [8]. The general aim of this paper is to propose a mathematical approach for capacity expansion planning. This model will determine where additional resources should be placed within airport terminal processes. Hence, the objective function of the proposed model is to minimise the cost of used resources and the total waiting time. A number of technical constraints exist.

The main contribution of this work is to provide a Capacity Expansion Planning Model (CEPM) for airports. Therefore, this paper has contributed to the body of knowledge by enabling two levels of planning, operational and strategic. In comparison with existing approaches, the new approach is more accurate because real waiting time can be identified and effects of uncertainty can be included. For example, Sun and Schonfeld [9] used mathematical functions, which are more approximate.

\section{Related Work}

This section analyses existing research conducted to address the issues of passenger flows within airports. The irregular flows occurring in airport terminal areas represent a significant management challenge, for instance, determining the number of service counters to open, and personnel allocation and reallocation issues [5-8, 10, 11]. A significant problem when studying passenger flow is capturing stochastic elements. This is because, as Guizzi et al. [12] argued, passengers behave differently inside airports according to their previous experiences. Thus, in order to assist decisionmakers at the airport terminal to address sudden and unforeseen congestion conditions, extensive research has been conducted on uncertainty. Yamada et al. [13] examined links between passenger behavior and facilities and identified several sources of congestion.

Additionally, Alodhaibi et al. [14] conducted their research using a simulation framework developed in [15] to investigate how the arrival pattern of passengers affects international terminal operations. The simulation outcomes provided a better understanding of the behavior of passenger airport access, which could lead to reduced waiting time and possible congestion by increasing the number of working stations (i.e., number of check-in counters) at peak times.

Safety concerns in recent times have caused many changes to security screening procedures and this impacts passenger throughput times. Previous research can be categorized according to related topic areas, including the significance of the security screening system in airport operations, the capacity of security screening areas, and dynamic system management [16-21]. For instance, Dorton and Liu [21] proposed the application of a DES for the security screening system coupled with a queuing. The aim is to analyze external factors influencing security screening operation efficiency and to identify the effect on throughput and cycle time.

In airport apron areas, a common optimization problem is gate assignment, which considers the minimization of passenger walking distance, from check-in to baggage claim area. Genç et al. [22] applied a heuristic to solve stochastic approaches to minimise the total duration of ungated flight. Similarly, Ding et al. [23] applied a Tabu Search metaheuristic to identify the problem when the number of aircraft exceeds the number of available gates. The two objective functions optimized were number of ungated aircraft and total walking distance. Finally, the research by Mota [24] is noteworthy as a mix of two models was applied to satisfy the different mandatory restricted policies related to airport terminal processing units, such as opening or closing check-in counters for each flight, check-in starting time, and load balance.

Capacity planning problems feature prominently in airport terminal research. For instance, Solak et al. [8] considered terminal operations to be a network system and used a multistage stochastic-integer linear programing model to determine the optimal capacity, taking into account optimal future expansion and desired LOS. The main objective was to minimise the maximum delay of each passageway and processing station by considering the variation in demand as a significant constraint. Also, Sun and Schonfeld [9] investigated uncertainties within the terminal. They found that facility performances are nonlinear functions. These functions are represented by delay level as a function of utilisation rates of capacity and demand fluctuations as indicated by uncertainties in traffic predictions. It is known that passenger departure flow is an important process for any airport facility because of the fixed departure time of flights. The same researchers $[25,26]$ go further by considering strategic airport facility planning under demand uncertainty and proposed a mixed-integer nonlinear program to determine when and where to adjust process capacity over a number of planning periods. Airport congestion and delay cost are approximated using some appropriate mathematical functions.

The novelty of our work lies in integrating a discrete event simulation to an optimization model for airport terminal capacity planning. Hence, undertaking such research is significant to identify a real waiting time and to know the effect of decision. Other models like Sun and Schonfeld's [9, 27], for instance, use mathematical functions, which are exact. Furthermore, in [26], the same authors used a discrete approximation technique when the model is solved where the mathematical relations in the formulation are accurate. Hence, a simulated annealing metaheuristic was applied to perform airport terminal capacity expansion and this may be the first application of such an approach to this domain. 


\section{Problem Description and Formulation}

This section defines the variables and parameters used in our capacity expansion model. The purpose of the model is to determine where additional resources should be placed in order to reduce the waiting costs. Each resource type has different costs, and a budget for total spending has been provided.

\subsection{Model Notation}

(i) Indices

$p, r, k, t, f:$ process, resource, passenger types, period, and shift.

(ii) Sets

$P, R, K, T, F$ : processes, resources, passenger types, periods, and shifts.

(iii) Parameters

$\bar{N}_{r, p}, \bar{N}_{r}$ : maximum number of resources of type $r$ in process $p$ and across all processes

$C_{r, p}$ : cost of providing a resource of type $r$ in process $p$

$V_{k, p}$ : cost incurred per unit of waiting time for passengers of type $k$ in process $p$

$B$ : total budget available for capacity expansion

$n_{r, p}$ : current number of resources of type $r$ in process $\mathrm{p}$

$\tau_{k, p}$ : expected time taken to serve passenger $k$ in process $p$.

(iv) Decision variables

$N_{r, p}$ : number of resources to open of type $r$ in process $p$

$W_{k, p}$ : total waiting time incurred in process $p$ for passengers of type $k$.

The model is as follows:

$$
\begin{array}{r}
\operatorname{minimize} \sum_{p} \sum_{r} C_{r, p} N_{r, p}+\sum_{k} \sum_{p} V_{k, p} W_{k, p} \\
\text { [resource cost }+ \text { cost of waiting], }
\end{array}
$$

subject to

$$
\begin{aligned}
& N_{r, p} \leq \bar{N}_{r, p}, \quad \forall p \in P ; \forall r \in R \quad \text { [upper bound], } \\
& \sum_{p} N_{r, p} \leq \bar{N}_{r}, \quad \forall r \in R \quad \text { [upper bound], } \\
& \sum_{r} C_{r, p} N_{r, p} \leq B, \quad \forall p \in P \quad \text { [budget constraints], } \\
& f_{r, p} \leq F_{r, p}, \quad \forall p \in P ; \forall r \in R \quad \text { [shift constraints], } \\
& N_{r, p} \geq 0, \quad \forall p \in P ; \forall r \in R \quad \text { [positivity], } \\
& W_{k, p}=\operatorname{SIMULATE}\left(N_{r, p}, \tau_{k, p}\right) \\
& \text { [calculation of waiting time via simulation]. }
\end{aligned}
$$

The objective function (1) has two components: (i) the cost of purchasing/acquiring additional resources of type $r$ in process $p$ and (ii) the total passenger waiting time converted to a dollar value. Constraints (2) and (3) ensure that the additional resources of type $r$ do not exceed the maximum number of resources. Constraint (4) restricts spending to a particular budget. Constraint (5) restricts the decision variable $N_{r, p}$ to be positive. The waiting time of passengers at different processes is a stochastic auxiliary variable and depends on the number of resources assigned and the processing time, which is a random variable. It is computed using simulation in equation (7) using the simulation model from $[11,15]$.

3.2. Simulated Annealing. To solve the proposed model, a metaheuristic approach is advocated as constraint (7) cannot be handled using mixed-integer programing, without the application of a simulation model. Of the different metaheuristics, simulated annealing was chosen. It is an effective and computationally fast search algorithm for solving hard optimization problems by Burdett and Kozan [27-29] and is well suited to probabilistic and nonlinear optimization problems. The algorithm is iterative and comprises two nested "for" loops. The outer loop controls and alters the "temperature" parameter $T$. In the inner loop, a specified number of solution refinements (a.k.a. perturbations) are entertained and evaluated. Refinements are accepted when they are explicitly better; otherwise, they are accepted/ rejected probabilistically according to the following function:

$$
P(\Delta f)=e^{-1 *(\Delta f / T)},
$$

where $\Delta f=f^{\prime}-f$ is the difference between the new solution $f^{\prime}$ and the current solution $f$. At every temperature, a selected number of perturbations are evaluated. SA requires several parameters (i.e., primary temperature, the cooling rate, the number of function evaluations at every temperature, and the final temperature). At early stages of the search, the temperature is high and many nonimproving moves are accepted. As the search progresses, the temperature is reduced and solutions are only accepted if a strict improvement occurs. With the slow reduction in temperature, worse solutions are accepted with less probability. The SA metaheuristic was implemented in $\mathrm{C}++$ and the simulation model was integrated to evaluate the waiting times. Preliminary numerical testing was performed to identify an appropriate starting temperature (see Figure 1). It is evident from the graph below that the best parameter values of this problem are as follows: temperature $(T)=15000$, cooling rate $(\alpha)=0.015$, and total number of iterations $=600$.

\subsubsection{Simulated Annealing Algorithm Description}

(1) Phase 1: Create Initial Solution. SA may be initialized with a randomly created solution or via some heuristic/constructive algorithm. However, because of the resource limitation constraints, some of the generated solutions will 

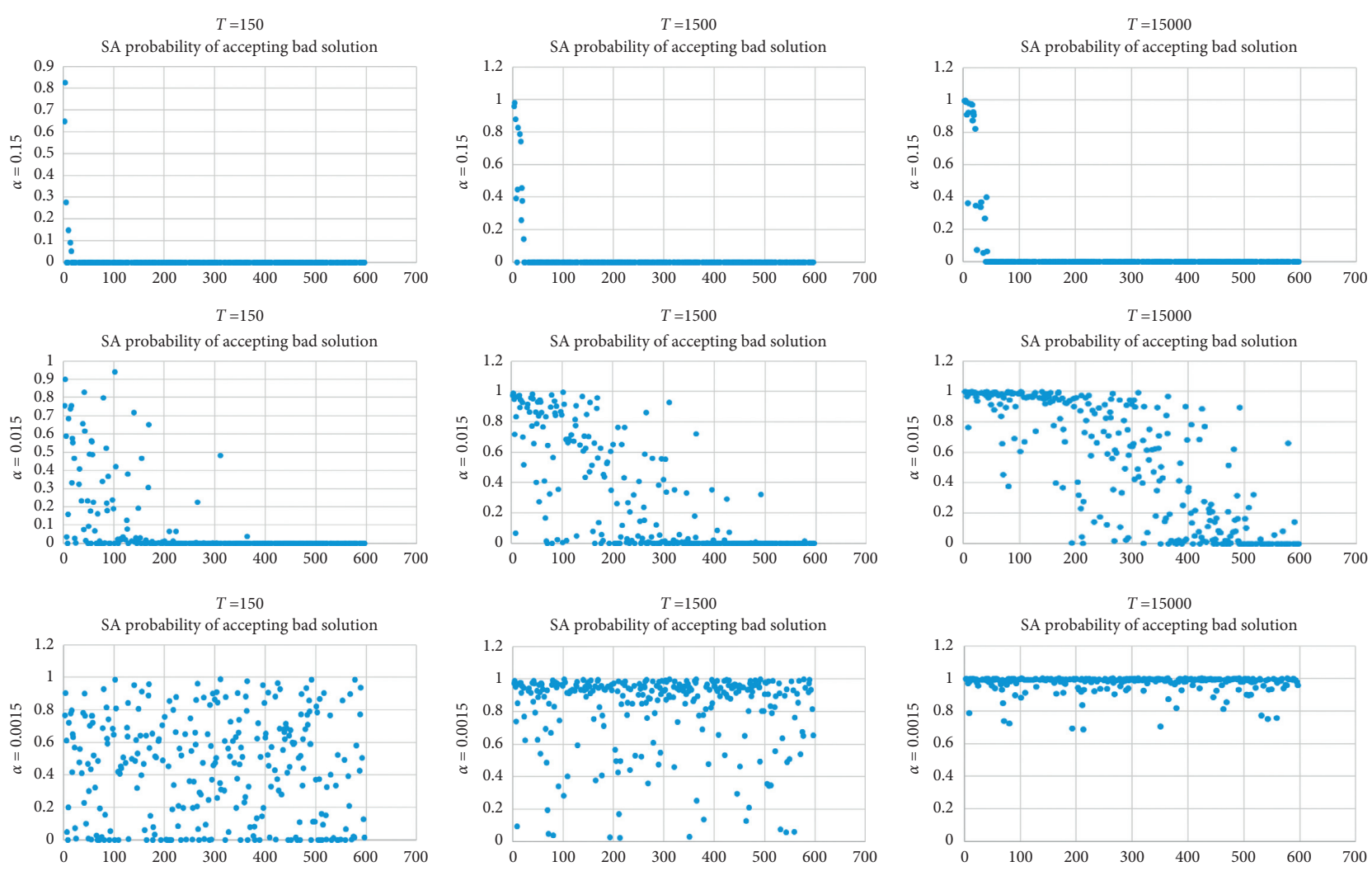

FIgURE 1: Selecting the best initial parameters.

not be feasible. The solution chromosome should simultaneously reflect two main characteristics:

(i) Number of resources for each process, such as checkin resources (i.e., economy and business counters), security screening resources, and immigration resources (i.e., common counters and SmartGates).

(ii) The number of assignment resources for each shift.

These solutions should be corrected via a corrective algorithm. Algorithm 1 is used to initialize a set of solutions.

(2) Phase 2: Perturbing a Solution. To create a new solution, it is necessary to perturb the current solution. There are many perturbation techniques that can be applied. In this article, a new solution is created by randomly changing the number of resources assigned to a randomly selected airport process. The creation of a new solution is performed by Algorithm 2.

(3) Phase 3: Assess New Solution. In this step, the goodness of the new solution is evaluated. Algorithm 3 demonstrates the assessment procedures. The generated solution will be simulated to measure a performance matrix, such as the average waiting time at each processing point. Also, the best cost will be selected by comparing it with the current cost.

(4) Phase 4: Stop Criteria. Finally, the condition of stopping the $\mathrm{SA}$ algorithm is based on the given maximum number of iterations. Algorithm 4 is an illustration of the main loop of the stopping criteria algorithm.

\section{Numerical Testing and Analysis}

In this section, the SA metaheuristic approach coupled with discrete event simulation is analyzed. In this numerical investigation, there are three types of process and five types of resources that were considered. Process type 1, the checkin process, has five separate lines, each with eight counters, two for business and six for economy. Process type 2 is security screening with five lanes. Process type 3 is immigration, which has eight common counters and 10 SmartGates. It is assumed that there are three periods during the day to which these processes are assigned to be operated. Figure 2 is a snapshot of the simulation outputs, for instance, the appropriate number of resources that need to be opened, the average waiting time that passengers spent at each process, and the cost of resources ending with the total cost.

The cost of waiting time is considered based on the given policy of acceptable queue time in a particular process. This is named the cost of inconvenience, as it exceeds given acceptable average waiting time. For example, passengers at the check-in process can be classified as business and economy, each with different queue time limits. Kazda and Caves [30] argued that the average waiting time should not be higher than 12 minutes for economy class and 3 minutes for business. The summary of input data used in this study is listed in Table 1.

Two different methods of creating new solutions were used to generate a starting solution. The first is creating a new solution randomly and the second is local search. The 


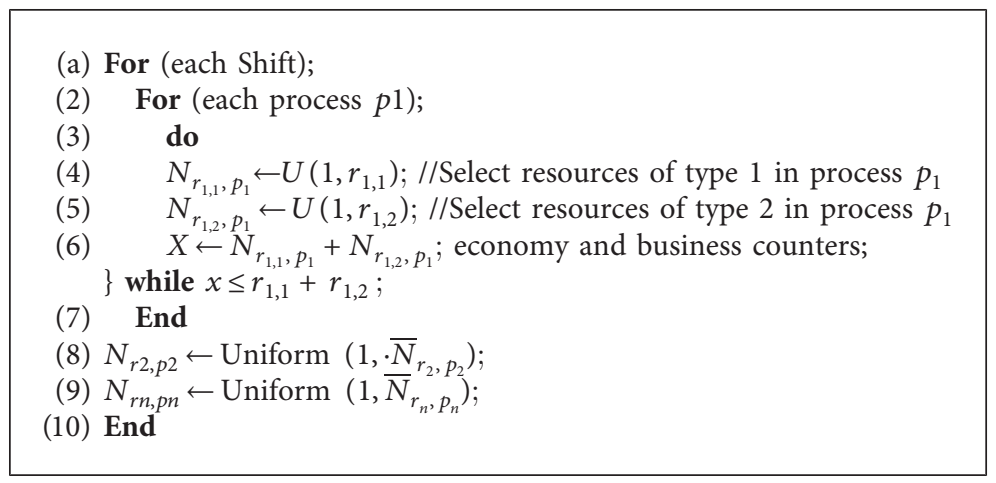

Algorithm 1: CreateSolution.

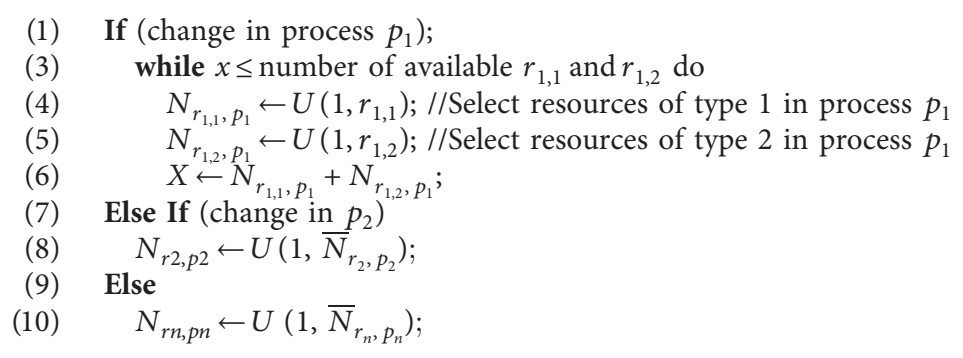

Algorithm 2: PerturbSolution.

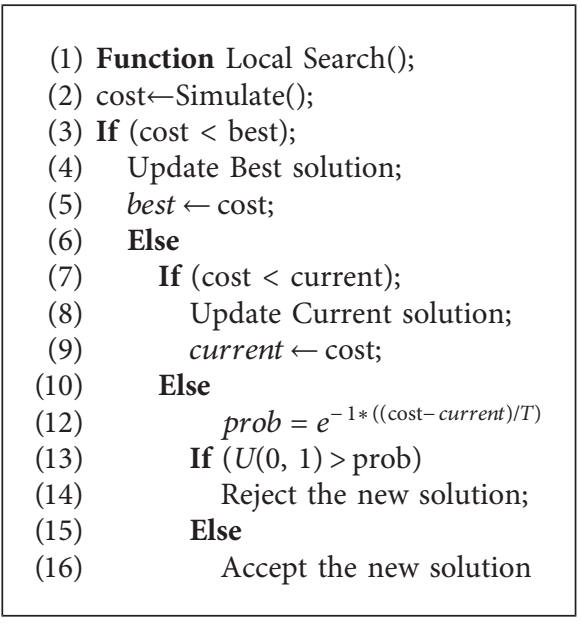

Algorithm 3: EvaluateSolution.

random search method initializes SA with a randomly created solution, while the local search initializes SA via constructive algorithm by changing one solution chromosome and then refinement by SA. For each method, 10 runs were repeated with the same parameters. The results of the runs are presented in Tables 2 and 3. The general parameters used for both methods are as follows: temperature $(T)=$ 15000 , cooling rate $(\alpha)=0.015$, and the maximum number of runs $=1500$.

The first column refers to the number of better solutions, where the average number of better solutions obtained by random search approach is 7.9 and the local search approach
(1) Parameter initialisation
(2) CreateSolution();
(3) Simulate();
(4) do
(5) PerturbSolution();
(6) EvaluateSolution();
(7) $\quad x \leftarrow x+1$;
(8) $\}$ while $(\mathrm{x}<$ max_iter $)$

Algorithm 4: Main loop.

is able to find 7.8 better solutions on average. The second column presents the total average waiting time spent in the airport terminal process. It is evident that local search provides lower waiting times compared with the random approach. It also has shorter run-time, with an average of 15.27 minutes compared to 21.05 minutes for the random approach. However, from the results presented in both tables, it can be clearly seen that the random search method reduces the objective function value by $12.11 \%$. The mean $\mu$ value of the objective function obtained from the random approach is $\$ 1998.3$, whereas $\mu$ of objective function value of the local search is $\$ 2256$.

From the 10 replications of both random search and local search, solution numbers 4 and 6 from the random search and local search were selected as the best solutions for two reasons. The first reason is that the value of the objective function is closer to the mean value of all the objective values. The second reason is that the chosen simulation runs 


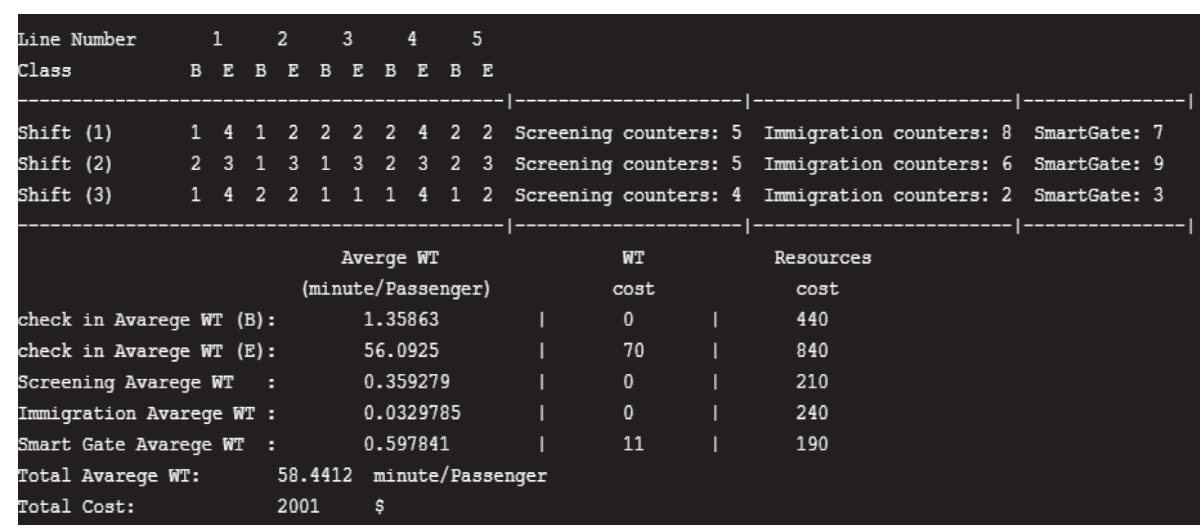

FIGURE 2: Snapshot of simulated annealing results.

TABLE 1: Summary of the input data.

\begin{tabular}{lc}
\hline Domain of the airport & Values \\
\hline Check-in & $20 \$$ \\
(i) Cost for opening new check-in counter & 12 minutes \\
(ii) The acceptable average waiting time for economy passengers & $15 \$$ \\
(iii) Cost for inconvenience at check-in for economy & 3 minutes \\
(iv) The acceptable average waiting time for business passengers & $25 \$$ \\
(v) Cost for inconvenience at check-in for business & $15 \$$ \\
Security screening & 5 minutes \\
(i) Cost for opening new security screening desk & $15 \$$ \\
(ii) The acceptable average time that normal passengers should wait & 2 minutes \\
(iii) Cost for inconvenience at security screening for normal passengers & $20 \$$ \\
(iv) The acceptable average time that diplomatic passengers should wait & $15 \$$ \\
(v) Cost for inconvenience at security screening for diplomatic passengers & $10 \$$ \\
\hline Immigration & \\
(i) Cost for opening new immigration desk & $20 \$$ \\
(ii) Cost for opening new SmartGate & $10 \$$ \\
(iii) The acceptable average waiting time at common counter & \\
(iv) Cost for inconvenience at common counters & \\
(v) The acceptable average waiting time at SmartGate & \\
(vi) Cost for inconvenience at SmartGate &
\end{tabular}

TABLE 2: Summary of simulated annealing results using random search technique.

\begin{tabular}{lcccc}
\hline & & \multicolumn{3}{c}{ Random search } \\
Run \# & \# of better solutions & Total average waiting time & Objective function value & Run-time (min) \\
\hline Run 1 & 6 & 118.73 & 2031 & 21.05 \\
Run 2 & 5 & 58.44 & 2001 & 21.16 \\
Run 3 & 10 & 126.39 & 2038 & 23.48 \\
Run 4 & 9 & 39.57 & 1973 & 23.43 \\
Run 5 & 6 & 64.74 & 1874 & 20.24 \\
Run 6 & 9 & 148.18 & 1913 & 17.36 \\
Run 7 & 8 & 112.714 & 1997 & 21.55 \\
Run 8 & 10 & 86.67 & 2052 & 17.36 \\
Run 9 & 7 & 127.8 & 2044 & 24.36 \\
Run 10 & 9 & 131.25 & 1998.3 & 21.054 \\
\hline$\mu$ & 7.9 & 101.4484 & 58.99160957 & 2.256671 \\
\hline
\end{tabular}

provide the minimum total average waiting time in the airport.

Figure 3 demonstrates the best solution given by the random search for the solution run number 4 . The optimal solution for this simulation run with regard to opening additional resources for check-in is summarized in Table 4. The average waiting time is 1.91 minutes for business-class passengers and 34.5 minutes for economy-class passengers. 
TABLE 3: Summary of simulated annealing results using local search technique.

\begin{tabular}{lcccc}
\hline & & \multicolumn{3}{c}{ Local search } \\
Run \# & \# of better solutions & Total average waiting time & Objective function value & Run-time (min) \\
\hline Run 1 & 7 & 38.88 & 2336 & 13.7 \\
Run 2 & 10 & 92.31 & 2195 & 14.02 \\
Run 3 & 9 & 80.49 & 2182 & 15.4 \\
Run 4 & 10 & 94.97 & 2042 & 15.48 \\
Run 5 & 8 & 22.25 & 2180 & 17.22 \\
Run 6 & 8 & 28.89 & 1994 & 12.22 \\
Run 7 & 8 & 72.6 & 2531 & 17.27 \\
Run 8 & 8 & 85.47 & 2638 & 12.21 \\
Run 9 & 4 & 86.45 & 2215 & 13.27 \\
Run 10 & 6 & 45.6 & 2256.3 & 15.27 \\
\hline$\mu$ & 7.8 & 64.82 & 186.49 & 2.88 \\
\hline
\end{tabular}

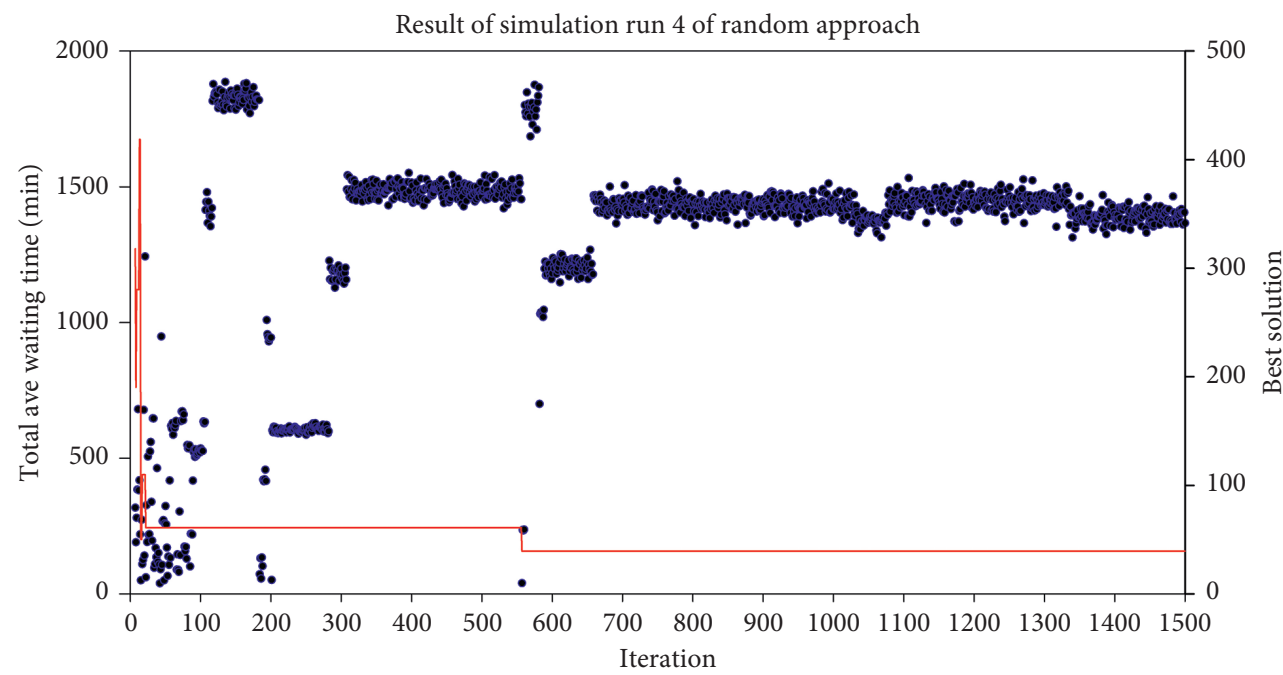

- Waiting_time for all runs

— Best solution

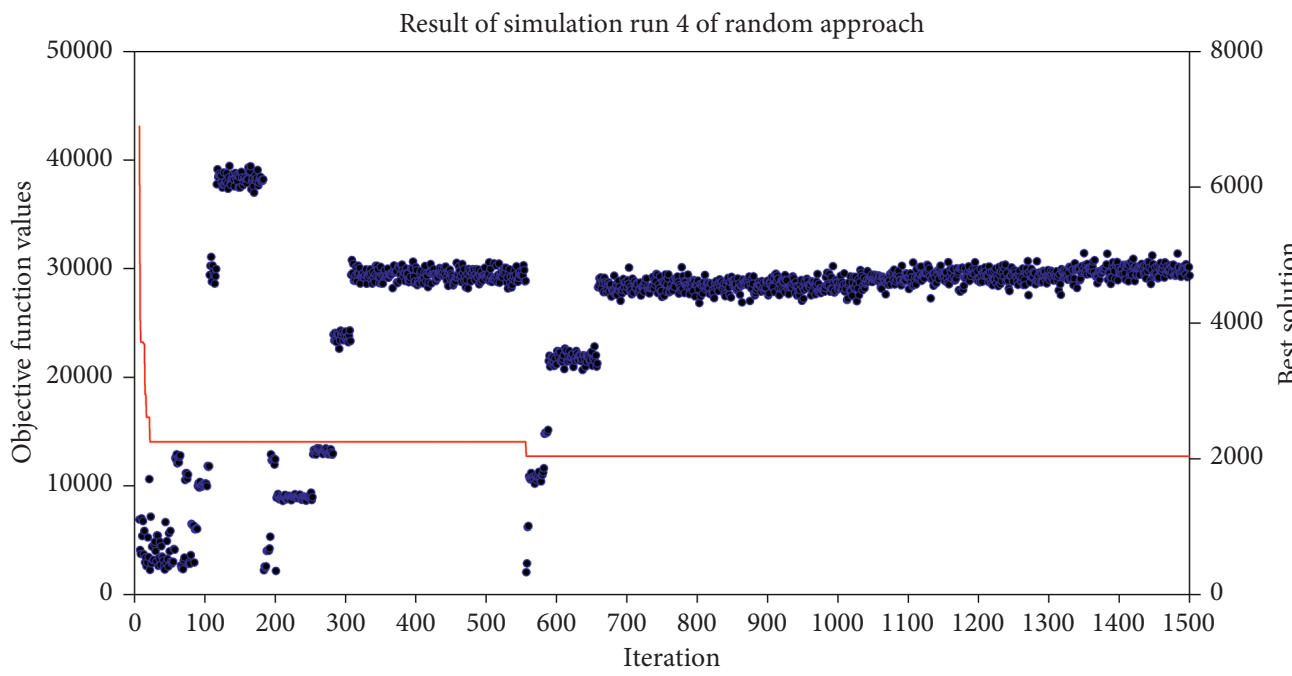

- Objective

- Best solution

FIGURE 3: SA optimisation results using the random method of creating new solutions. 
TABLE 4: Check-in additional resource results using the random technique.

\begin{tabular}{lcccccccccc}
\hline & \multicolumn{2}{c}{ Line 1 } & \multicolumn{2}{c}{ Line 2 } & \multicolumn{2}{c}{ Line 3 } & \multicolumn{2}{c}{ Line 4} & \multicolumn{2}{c}{ Line 5 } \\
& Business & Economy & Business & Economy & Business & Economy & Business & Economy & Business & Economy \\
\hline Shift 1 & 1 & 4 & 2 & 3 & 2 & 4 & 1 & 4 & 1 & 4 \\
Shift 2 & 2 & 2 & 1 & 1 & 1 & 1 & 1 & 2 & 2 \\
Shift 3 & 2 & 4 & 1 & 6 & 1 & 6 & 1 & 2 & 2 \\
\hline
\end{tabular}
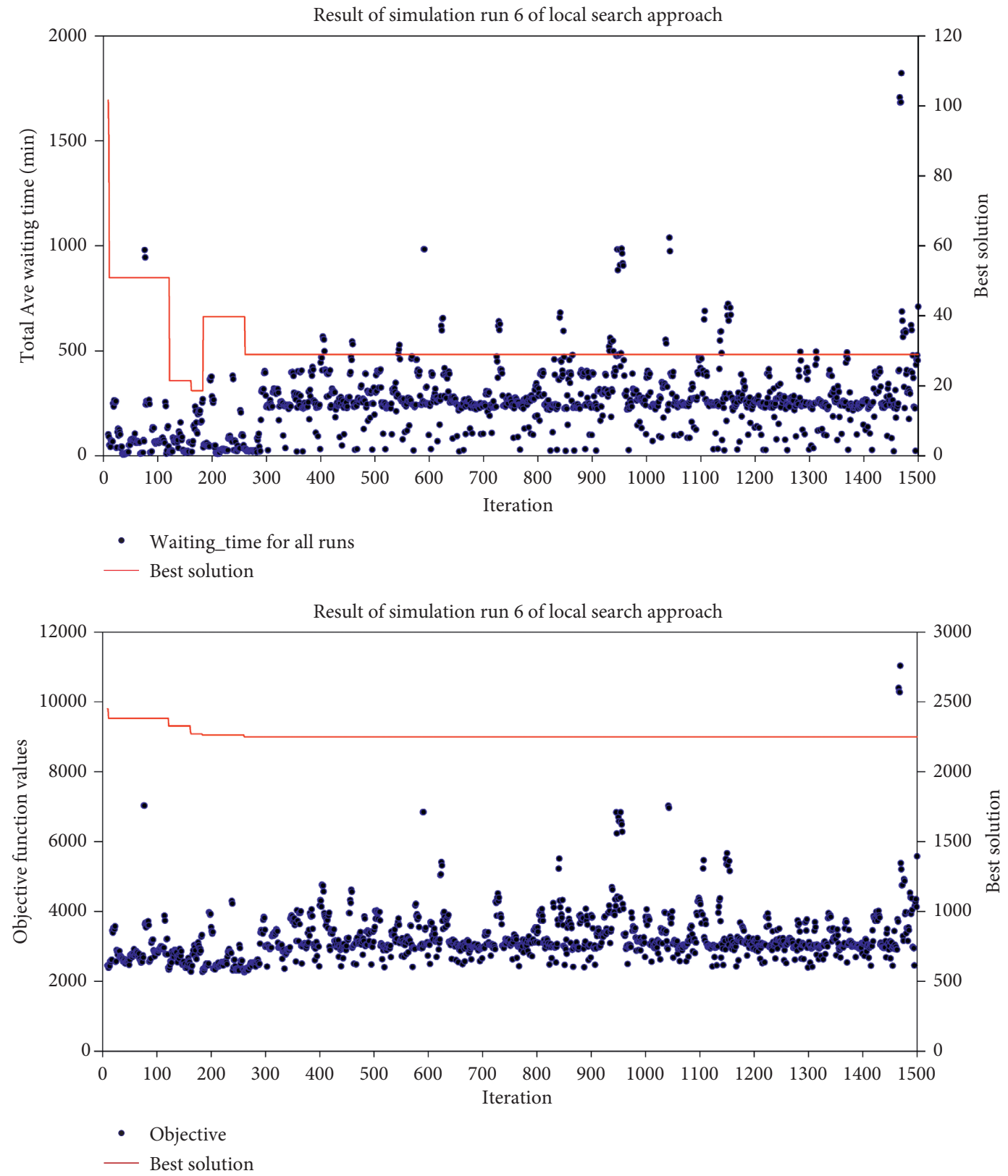

FIGURE 4: Optimisation results using the method of creating new solution using local technique.

For the security screening checkpoints, the opening resources are 5, 2, and 3 control checkpoints for shift 1 , shift 2 , and shift 3 , respectively, having an average waiting time of 3.04 minutes. The opening common immigration process is 4, 3, and 5 counters, while for SmartGates there are 8, 9, and 7 kiosks for the three shifts, having average waiting times of 0.11 and 0.02 minutes for the common immigration desks and SmartGate kiosk, respectively. The total cost of opening all resources is $\$ 2038$ given that the total average time spent in the queues is 39.57 minutes.

Figure 4 illustrates the optimal solution provided by the SA algorithm using local search for creating a new solution. In this simulation run, the solution is characterised from the total cost of $\$ 2250$ given that the total average waiting time 
TABLE 5: Check-in additional resource results using local technique.

\begin{tabular}{lcccccccccc}
\hline & \multicolumn{2}{c}{ Line 1 } & \multicolumn{2}{c}{ Line 2 } & \multicolumn{2}{c}{ Line 3 } & \multicolumn{2}{c}{ Line 4} & \multicolumn{2}{c}{ Line 5 } \\
& Business & Economy & Business & Economy & Business & Economy & Business & Economy & Business & Economy \\
\hline Shift 1 & 1 & 4 & 2 & 3 & 3 & 5 & 2 & 5 & 1 & 5 \\
Shift 2 & 2 & 7 & 1 & 2 & 1 & 3 & 4 & 3 & 1 \\
Shift 3 & 2 & 1 & 2 & 2 & 2 & 2 & 4 & 1 & 2 \\
\hline
\end{tabular}

spent in the system is 28.89 minutes. The optimal solution for this simulation run is opening a check-in resource based on the detailed information listed in Table 5. By adding this resource, the average waiting time at the check-in process will be 1.56 minutes for business-class passengers and 7.03 minutes for economy-class passengers, reductions of $20.17 \%$ for business-class passengers and $132.29 \%$ for economyclass passengers compared with the random search method.

For the process of security screening checkpoints, the best solution can be found when opening 4,4 , and 5 security control checkpoints for shift 1 , shift 2, and shift 3, respectively. By opening these numbers of resources at the security screening process, the average waiting time is 7.92 minutes. Finally, the immigration process should open 5, 4, and 3 common immigration counters and 10, 10, and 9 SmartGates for the three shifts, in order to get the optimal solution, having the average waiting of 0.10 minutes and 0.012 minutes for the common counters and SmartGates, respectively.

\section{Conclusion}

This paper has discussed the development of a mathematical approach to perform capacity planning in airport terminals. The objective of the model is determining where additional resources should be opened to decrease the cost of time spent in the queues of the airport terminal. Since the proposed problem is probabilistic and nonlinear, a metaheuristic approach is advocated. The waiting times are computed using discrete event simulation and those times are used in the objective function. Two different approaches for creating new solutions were used in this study. The first one is creating a new solution using the random technique and the other is creating a new solution by using the local search technique. The random technique decreased the objective function value by $12.11 \%$. It also has shorter runtimes, with average of 15.27 minutes compared to 21.05 minutes for the random approach.

The developed model can be more accurate because the effect of decision can be known and real time can be identified. It also can provide strategic planning, while the proposed simulation model can be used for the operational planning level. This work has contributed to the body of knowledge by enabling two levels of planning, operational and strategic.

On the other hand, the lack of access to the detailed data related to operational facilities due to the recent strict regulations with respect to security issues resulted in some difficulties in developing the modelled passengers' flows within international terminal. Because of these difficulties, this model has been simplified by, firstly, utilizing available data collected by previous research, and, secondly, making an assumption where needed.

\section{Data Availability}

No data were used to support this study.

\section{Conflicts of Interest}

The authors declare that they have no conflicts of interest.

\section{Authors' Contributions}

Dr. Sultan Alodhaibi mainly undertook this research as a $\mathrm{Ph} . \mathrm{D}$. student and formulated and conducted the experimental design. He designed, developed, and tested the simulation models and wrote the main manuscript. Dr. Robert L. Burdett provided necessary guidance for mathematical optimisation of simulation models and interpretation of the simulation results. Professor Prasad K. D. V. Yarlagadda conceived the project and got necessary infrastructure and funding for undertaking this research. He also planned and helped in design of simulation model and related experiments and supervised and oversaw the full project from its inceptual stage to final stages of delivery. All authors reviewed and commented on the manuscript.

\section{Acknowledgments}

This research was supported by the Australian Research Council's Linkage Project "Improving Productivity and Efficiency of Australian Airports" (140100282). The authors would like to acknowledge QUT High Performance Computing (HPC) for providing the computational resources for this research.

\section{References}

[1] C. Barnhart, D. Fearing, A. Odoni, and V. Vaze, "Demand and capacity management in air transportation," EURO Journal on Transportation and Logistics, vol. 1, no. 1-2, pp. 135-155, 2012.

[2] R. D. Neufville and A. Odoni, Airport Systems: Planning, Design and Management, McGraw-Hill Professional, New York, NY, USA, 2003.

[3] K. G. Zografos and M. A. Madas, "Development and demonstration of an integrated decision support system for airport performance analysis," Transportation Research Part C: Emerging Technologies, vol. 14, no. 1, pp. 1-17, 2006.

[4] I. E. Manataki and K. G. Zografos, "A generic system dynamics based tool for airport terminal performance analysis," Transportation Research Part C: Emerging Technologies, vol. 17, no. 4, pp. 428-443, 2009. 
[5] P. P.-Y. Wu and K. Mengersen, "A review of models and model usage scenarios for an airport complex system," Transportation Research Part A: Policy and Practice, vol. 47, pp. 124-140, 2013.

[6] S. Alodhaibi, R. L. Burdett, and P. K. Yarlagadda, "A model to simulate passenger flow congestion in airport environment," International Journal of Engineering \& Technology, vol. 7, no. 4, pp. 6943-6946, 2018.

[7] R. D. Neufville and A. R. Odoni, Airport Systems: Planning, Design, and Management, McGraw-Hill Education, New York, NY, USA, 2013.

[8] S. Solak, J.-P. B. Clarke, and E. L. Johnson, "Airport terminal capacity planning," Transportation Research Part B: Methodological, vol. 43, no. 6, pp. 659-676, 2009.

[9] Y. Sun and P. Schonfeld, "Stochastic capacity expansion models for airport facilities," Transportation Research Part B: Methodological, vol. 80, pp. 1-18, 2015.

[10] P. Fonseca, J. Casanovas, and X. Ferran, "Passenger flow simulation in a hub airport: an application to the Barcelona international airport," Simulation Modelling Practice and Theory, vol. 44, pp. 78-94, 2014.

[11] S. Alodhaibi, R. L. Burdett, and P. K. D. V. Yarlagadda, "A framework for sharing staff between outbound and inbound airport processes," Mathematics, vol. 8, no. 6, p. 895, 2020.

[12] G. Guizzi, T. Murino, and E. Romano, "A discrete event simulation to model passenger flow in the airport terminal," in Proceedings of the 11th WSEAS International Conference on Mathematical Methods and Computational Techniques in Electrical Engineering (MMACTEE'09), Athens, Greece, September 2009.

[13] H. Yamada, K. Ohori, T. Iwao et al., "Modeling and managing airport passenger flow under uncertainty: a case of Fukuoka airport in Japan," in Social Informatics, pp. 419-430, SpringerVerlag, Cham, Switzerland, 2017.

[14] S. Alodhaibi, R. L. Burdett, and P. K. D. V. Yarlagadda, "Impact of passenger-arrival patterns in outbound processes of airports," Procedia Manufacturing, vol. 30, pp. 323-330, 2019.

[15] S. Alodhaibi, R. L. Burdett, and P. K. Yarlagadda, "Framework for airport outbound passenger flow modelling," Procedia Engineering, vol. 174, pp. 1100-1109, 2017.

[16] K. Leone and R. Liu, "Improving airport security screening checkpoint operations in the US via paced system design," Journal of Air Transport Management, vol. 17, no. 2, pp. 6267, 2011.

[17] J. V. Boekhold, A. Faghri, and M. Li, "Evaluating security screening checkpoints for domestic flights using a general microscopic simulation model," Journal of Transportation Security, vol. 7, no. 1, pp. 45-67, 2014.

[18] A. Kierzkowski and T. Kisiel, "Simulation model of security control system functioning: a case study of the Wroclaw airport terminal," Journal of Air Transport Management, vol. 64, no. Part B, pp. 173-185, 2016.

[19] J. Skorupski and P. Uchroński, "Managing the process of passenger security control at an airport using the fuzzy inference system," Expert Systems with Applications, vol. 54, pp. 284-293, 2016.

[20] A. Kierzkowski and T. Kisiel, "Evaluation of a security control lane with the application of fuzzy logic," Procedia Engineering, vol. 187, pp. 656-663, 2017.

[21] S. Dorton and D. Liu, "Effects of baggage volume and alarm rate on airport security screening checkpoint efficiency using queuing networks and discrete event simulation," Human
Factors and Ergonomics in Manufacturing \& Service Industries, vol. 26, no. 1, pp. 95-109, 2016.

[22] H. M. Genç, O. K. Erol, İ. Eksin, M. F. Berber, and B. O. Güleryüz, "A stochastic neighborhood search approach for airport gate assignment problem," Expert Systems with Applications, vol. 39, no. 1, pp. 316-327, 2012.

[23] H. Ding, A. Lim, B. Rodrigues, and Y. Zhu, "The over-constrained airport gate assignment problem," Computers \& Operations Research, vol. 32, no. 7, pp. 1867-1880, 2005.

[24] M. M. Mota, "Check-in allocation improvements through the use of a simulation-optimization approach," Transportation Research Part A: Policy and Practice, vol. 77, pp. 320-335, 2015.

[25] Y. Sun and P. M. Schonfeld, "Capacity investment model for airport facilities under demand uncertainty," Journal of Advanced Transportation, vol. 50, no. 8, pp. 1896-1911, 2016.

[26] Y. Sun and P. M. Schonfeld, "Coordinated airport facility development under uncertainty," Transportation Research Record: Journal of the Transportation Research Board, vol. 2603, no. 1, pp. 78-88, 2017.

[27] R. L. Burdett and E. Kozan, "Sequencing and scheduling in flowshops with task redistribution," Journal of the Operational Research Society, vol. 52, no. 12, pp. 1379-1389, 2001.

[28] R. L. Burdett and E. Kozan, "An integrated approach for earthwork allocation, sequencing and routing," European Journal of Operational Research, vol. 238, no. 3, pp. 741-759, 2014.

[29] R. L. Burdett, P. Corry, P. K. D. V. Yarlagadda, C. Eustace, and S. Smith, "A flexible job shop scheduling approach with operators for coal export terminals-a mature approach," Computers and Operational Research, vol. 104, pp. 15-36, 2019.

[30] A. Kazda and R. E. Caves, Airport Design and Operation, Emerald Group Publishing Limited, Bingley, UK, 2015. 